\title{
CUESTIONES DE MÉTODO: LA GRILLA DE INTELIGIBILIDAD EPOCAL-TOPOLÓGICA Y LA LÓGICA ESTRATÉGICA DE LAS CORRELACIONES Y SISTEMAS DE DOMINANTES EN LA HISTORIA POLÍTICA DE LA GUBERNAMENTALIDAD EN LOS CURSOS DE MICHEL FOUCAULT.
}

[Questions of method: The topological-epochal grid of intelligibility and the strategic logic of correlations and systems of dominants in the political history of governmentality in Michel Foucault's courses.]

Resumen: En este artículo se abordarán críticamente las hipótesis de lectura que Bidet y Collier proponen sobre el segundo Foucault con el objetivo de determinar en qué aspecto son pertinentes y bajo qué condiciones se pueden articular entre sí. Ello nos permitirá delinear una estrategia de interpretación que no sólo tome a los cursos como una serie, sino que también explique el tipo de la relación que guardan entre sí soberanía, disciplina y seguridad, pues de ello depende la posibilidad de comprensión del proyecto de una historia política de la gubernamentalidad.

Palabras clave: Grilla epocal-topológica - Lógica estratégica - historia de la gubernamentalidad Foucault.

Abstract: In this paper I propose a critical view about the Bidets and Colliers' hypothesis refers to the
second Foucault, in order to determine their relevant aspects and the conditions in wich they can be
connected from each other. It will allow to provide a strategic interpretation not only considering the
classes as a series, but also to explain what kind of relationship it could be determined between the
sovereignty, discipline and security as theoretical categories. I stand that this perspective provides the
possibility to understand the project of a political history of governmentality. Keywords: Topological-epochal grid of intelligibility - Strategical logic - History of governmentality Foucault.

\section{Introducción}

Suele afirmarse que la obra de Michel Foucault debe clasificarse en tres períodos. El segundo de los cuales abarcaría la década del ' 70 y tendría por objeto de estudio a los dispositivos de poder y el tipo de sujeto que le es correlativo. Este período estaría marcado por el ingreso de Foucault al Collège de France y, por lo tanto, comprendería no sólo los libros publicados y las conferencias, entrevistas y artículos recogidos en los Dits et Écrits, sino también los cursos dictados en dicha institución.

\footnotetext{
${ }^{I}$ Doctor en Ciencias Sociales por la Facultad de Ciencias Sociales (UBA). Profesor de 'Filosofía' por la Facultad de Filosofía y Letras (UBA). Docente de la Universidad Nacional de La Matanza (UNLaM) y de la Universidad de Buenos Aires (UBA). Investigador del Departamento de Derecho y Ciencia Política de UNLaM. Director del Proyecto de Investigación CYTMA2: "El concepto foucaulteano de "Política" en la segunda mitad de la década del '70: el lugar del "derecho" entre la guerra, el gobierno y la resistencia".
} 
Simultáneamente, suele considerarse a El orden del discurso como el comienzo de la articulación entre el estudio del saber con los dispositivos de poder. Por otra parte, suele afirmarse que Vigilar y Castigar, del año 1975, y La voluntad de saber, de 1976, marcan, respectivamente, la culminación de una serie de indagaciones y el inicio de otras. Así, mientras que el primer libro es el resultado de las investigaciones que ocupan a Foucault durante la primera mitad de la década -y que giran en torno de las tecnologías disciplinarias y el estudio de lo que denomina la "microfísica del poder"-, el segundo libro no sólo es presentado por el autor como el primero de una serie que se propone abordar la cuestión de la Historia de la sexualidad, sino que es -especialmente desde su último capítulo- el comienzo de una indagación sobre lo que Foucault denomina 'biopolítica' y que constituirá el trasfondo de las exploraciones llevadas a cabo durante la segunda mitad de la década.

Por un lado, en El poder psiquiátrico, el curso de 1973, Foucault señalaba que para analizar las relaciones de poder se debía pasar del estudio de la "macrofísica de la soberanía" al de la "microfísica del poder disciplinario"2. Por otro, en un giro complementario a este paso hacia las tácticas locales de poder, en el año 1976 Foucault emprende, a través del concepto de biopolítica, una serie de investigaciones sobre las estrategias globales de poder ${ }^{3}$. De esta forma, el segundo período de la obra se dividiría a su vez en dos momentos que abarcan la primera y la segunda mitad de la década.

En consecuencia, un análisis del período debe tomar en cuenta las diferentes interconexiones que existen entre los tres registros en que fue y aún continúa siendo publicada la obra de Michel Foucault, i.e. los libros publicados, los cursos dictados y las conferencias, entrevistas, intervenciones públicas y periodísticas recogidas mayormente en Dits et Écrits, a las que en la actualidad comienzan a sumarse los "archivos Foucault". En efecto, la relación que guarda Vigilar y castigar con los cursos precedentes y con los Dits et Écrits no puede ser analogada a la que tiene La voluntad de saber con los cursos, conferencias y escritos posteriores. En este sentido, respecto de la relación entre los cursos y los libros publicados, Venn y Terranova marcan un desfasaje a tener en cuenta en la producción foucaultiana de la época ${ }^{4}$. Los

\footnotetext{
${ }^{2}$ FOUCAULT, M. El poder psiquiátrico. Buenos Aires: Fondo de Cultura Económica, 2005, p. 44.

${ }^{3}$ Véase FOUCAUlT, M. Defender la sociedad. Buenos Aires, Fondo de Cultura Económica: 2000; y FOUCAULT, M. Historia de la sexualidad I: La voluntad de saber. México: Siglo Veintiuno editores, 2000.

${ }^{4}$ Véase VENN, C. y TERRANOVA, T. "Introduction. Thinking after Michel Foucault", Theory, Culture \& Society, 2009, Vol. 26(6):1-11.
} 
comentadores sostienen que los cursos deben organizarse, a su vez, en las tres series siguientes: la primera, que abarcaría los cursos dictados entre 1971 y 1974, tendría como eje la normalización y el poder disciplinario, y habría dado lugar a los libros sobre las instituciones penales y el dispositivo disciplinario (Vigilar y Castigar) y sobre el dispositivo de sexualidad (La Voluntad de Saber); la segunda, que incluiría los cursos dictados entre 1976 y 1979, giraría en torno de la genealogía de la biopolítica y su vínculo, por un lado, con la guerra de razas y, por el otro, con la economía política, el dispositivo de seguridad, la gubernamentalidad y el liberalismo, y que no habría tenido como correlato la publicación de los resultados de esta investigación en ningún libro, salvo la inclusión de la cuestión biopolítica en el último capítulo de La Voluntad de Saber, publicada al inicio de la preocupación foucaultiana sobre este problema ${ }^{5}$; por último, estaría la serie compuesta por los cursos sobre la ética grecorromana y la estética de la existencia y la parrhesía de la que habrían resultado los dos últimos libros de Foucault, a saber el segundo y el tercer volumen de Historia de la Sexualidad: El Uso de los Placeres y La inquietud de sí, respectivamente.

Si se toma en cuenta la organización de la obra en tres períodos y de los cursos en tres series salta a la vista, entonces, la dificultad en torno del estatus y de la relación entre sí y con el resto de la obra de los cursos sobre la biopolítica, la economía política y la gubernamentalidad. Si bien no es el objetivo de este artículo determinar el estatus de estos cursos ni hacer una interpretación de conjunto de la obra foucaultiana, dos observaciones al respecto son pertinentes para nuestro abordaje. En primer lugar, este desfasaje -i.e. la ausencia de libros publicados como resultado de las investigaciones que ocupan la segunda serie de los cursos- realza la importancia tanto de los cursos que la componen, cuanto de las entrevistas, conferencias y demás escritos del período. En segundo lugar, y como corolario de lo anterior, es importante -para brindar una interpretación plausible de esa segunda serie- determinar la relación que guardan estos cursos entre sí y con algunas de las intervenciones recogidas en Dits et Écrits. ${ }^{6}$ Para ello, proponemos una grilla de inteligibilidad epocal-topológica que apoyada en una

\footnotetext{
${ }^{5}$ Para ser exactos, la problemática de la biopolítica es planteada por primera vez en el año 1974 en una conferencia en Río de Janeiro. Véase FOUCAULT, M. "Nacimiento de la medicina social", en: Estrategias de poder. Obras esenciales, Volumen II. Barcelona: Paidós, 1999, p. 366.

${ }^{6}$ Véase FOUCAULT, M. Dits et Écrits II. Paris: Gallimard, 2001.
} 
lógica estratégica permita interpretarlos como una serie en la que se aborda el problema de una historia política de la gubernamentalidad. ${ }^{7}$

Sin embargo, Judith Revel establece una ruptura radical entre el curso de 1976 en el que se exploraría la hipótesis Nietzsche y el de 1978 donde emergería el concepto de gubernamentalidad como grilla de inteligibilidad de las relaciones de poder. ${ }^{8}$ Por su parte, Jacques Bidet, desde una perspectiva marxista, establece la ruptura entre el curso de 1976 y el de 1978 a partir de la innovación que introduce el concepto de gubernamentalidad pero ya no en relación con la hipótesis Nietzsche, como apuntaba Revel, sino respecto del vínculo entre Foucault y Marx. ${ }^{9}$ De este modo, según Bidet, entre los años 1971 y 1976 el discurso de Foucault estuvo atravesado por los conceptos de 'burguesía', 'proletariado' y 'clases sociales', a la vez que en el centro del mismo estaba la cuestión de la lucha de clases y, en última instancia, la de saber si la revolución, y cuál, valía la pena. A pesar de esto, durante este período Foucault se habría abocado al estudio de técnicas sectoriales de poder manteniéndose en los márgenes de "la gran política", es decir, de la "tecnología del poder del Estado". Sería, entonces, a partir de los cursos de 1978 y 1979 que el filósofo francés se abocaría a la cuestión de la política económica y social a través de los conceptos de gubernamentalidad y de resistencia que le serán correlativos. Allí, según Bidet, los caminos de Foucault se cruzarían con los de Marx. ${ }^{10}$ En un sentido bastante diferente Stephen Collier en su artículo Topologies of power explica la ruptura que introduciría el curso Seguridad, territorio, población respecto de Defender la sociedad a partir de lo que denomina el "análisis topológico del poder". ${ }^{11}$ En oposición a lo que designa como la interpretación dominante del período que aquí nos interesa -que consistiría en señalar la emergencia de la cuestión biopolítica en el curso de 1976 como un modo de extender los análisis con sus herramientas conceptuales hacia el Estado como un nuevo objeto-, el comentador señala que este tipo de análisis oscurece cierta modificación importante ocurrida en los cursos de los años 1978 y 1979- del método y del diagnóstico foucaultiano.

\footnotetext{
7 Véase CASTRO-GÓMEZ, S. Historia de la gubernamentalidad. Razón de Estado, liberalismo y neoliberalismo en Michel Foucault. Bogotá: Siglo del Hombre Editores, 2012.

${ }^{8}$ Véase REVEL, J. Dictionnaire Foucault. París: Ellipses, 2008.

${ }^{9}$ Véase BIDET, J. "Foucault y el liberalismo: Racionalidad, revolución, resistencia", Argumentos, Año 19 No. 52, Septiembre-Diciembre, 2006, pp. 1-27

${ }^{10}$ Véase BIDET, J. "Foucault y el liberalismo: Racionalidad, revolución, resistencia", Argumentos, Año 19 No. 52, Septiembre-Diciembre, 2006, p. 12.

11 COLLIER, S. "Topologies of Power. Foucault's Analysis of Political Government beyond 'Governmentality”, Theory, Culture \& Society, Vol. 26(6), 2009, p. 79.
} 
Según Bidet el tránsito del análisis del poder en términos de lucha de clases a uno centrado en el concepto de gubernamentalidad supondría el paso hacia la forma discursiva del "gran relato" a partir de la constatación de que vivimos en la "era de la gubernamentalidad" y de que Foucault se dirige hacia el estudio de la cuestión de "la totalidad social, considerada en términos de la "racionalidad occidental". ${ }^{12}$ Por el contrario, para Collier el mismo desplazamiento daría lugar a una interpretación opuesta punto por punto a la de Bidet. Mientras que, de acuerdo a Collier, en Defender la sociedad, la exploración del Estado y del gobierno político estaría marcada por un estilo de diagnóstico que enfatizaría "la sistematicidad, la coherencia funcional y el alcance totalizante de las formas de poder" ${ }^{\prime 1}$, en los cursos posteriores tal diagnóstico operaría a través de un análisis topológico -que buscaría examinar los ‘patrones de correlación' en los cuales ciertos elementos heterogéneos como las técnicas y las tecnologías de poder serían configurados, redesplegados y recombinados-. Así, Foucault se desplazaría desde una tendencia a formular diagnósticos globales acerca de las relaciones de poder diagnósticos orientados a resaltar una única lógica (de soberanía, disciplina o normalización) que configuraría una época dada- hacia una explicación que se centraría en el estudio de las formas contingentes de correlación de elementos, a través una lógica estratégica que no implicaría ninguna necesidad o coherencia interna. En este sentido, de acuerdo con Collier, el "gran relato" foucaultiano se diversificaría a partir de 1978 en una multiplicidad de diagnósticos específicos en los cuales el pensamiento estaría concebido como una práctica situada de reflexión crítica. ${ }^{14}$

Por su parte, en las antípodas de estos planteos, Couze Venn propone -con el fin de hacer una "genealogía transcolonial del presente"- una lectura de conjunto de los tres

12 BIDET, J. "Foucault y el liberalismo: Racionalidad, revolución, resistencia”, Argumentos, Año 19 No. 52, Septiembre-Diciembre, 2006, p. 13.

13 COLLIER, S. "Topologies of Power. Foucault's Analysis of Political Government beyond 'Governmentality”, Theory, Culture \& Society, Vol. 26(6), 2009, p. 79.

${ }^{14}$ Como resultará evidente a cualquier lector especializado en la obra de Michel Foucault, las recientes interpretaciones opuestas de Bidet y Collier no hacen sino retomar y reploblematizar la cuestión planteada por Michael Donnelly en ocasión del Encuentro Internacional organizado en París en enero de 1988 por la Association pour le Centre Michel Foucault. Allí el expositor apuntaba a señalar la tensión y el salto argumentativo que atravesaría la obra de Foucault desde el comienzo de los estudios genealógicos de la prisión. Como allí se afirma: "el problema sobre el cual quieren llamar la atención estas observaciones es el de la irreductible diferencia que hay entre los argumentos genealógicos y los argumentos 'epocales' de Foucault; de ahí la ambigüedad que nace desde el momento en que Foucault pasa de una línea de argumentación a otra", DONNELLY, M. "Sobre los diversos usos de la noción de biopoder", en: Balbier, E., Deleuze, G. et al., Michel Foucault filósofo. Barcelona: Gedisa, 1999, p. 197. Nuevamente, la lectura de Donnelly se apoya en la idea del abandono y el reemplazo para sostener que los argumentos genealógicos serían sustituidos por argumentos epocales "más débiles”. Como también resultará evidente, nuestra grilla epocal-topológica no pretende desambiguar esta relación de tensión, sino pensar a partir de ella, más allá de la lógica del abandono y el reemplazo. 
cursos para demostrar que hay dos genealogías superpuestas a través de ellos: por un lado, aquella que se ocupa del desplazamiento en la forma del poder en Europa y que lleva desde la forma de poder soberano y el discurso de la guerra de razas previa al siglo XVIII, pasando por el análisis del poder disciplinario y el pastoral después de Westfalia, para terminar con el estudio de la gubernamentalidad biopolítica moderna en el siglo XIX y finalmente con el de la Vitalpolitik medioambiental de lo social en la actualidad; por el otro, la genealogía de la economía política desde el mercantilismo en los siglos XVI, XVII y XVIII, pasando por la fisiocracia en el siglo XVIII, hasta llegar a la emergencia del liberalismo en el siglo XIX y su reconfiguración como ordo- y neoliberalismo en el siglo XX. ${ }^{15}$

En este artículo se ahondará en las hipótesis de lectura de Bidet y de Collier, retomando algunos aspectos de la de Venn, con el objetivo de determinar en qué medida son pertinentes y bajo qué condiciones se pueden articular entre sí. Ello nos permitirá delinear una estrategia de lectura que no sólo tome a los cursos como una serie, sino que también explique el tipo de la relación que guardan entre sí, pues de ello depende la posibilidad de comprensión del proyecto de una historia política de la gubernamentalidad.

\section{El "gran relato" foucaulteano ${ }^{16}$.}

Jacques Bidet propone una interpretación del pensamiento de Foucault que desligándolo de la corriente liberal busca comprender sus problematizaciones como inextricables de los análisis marxianos. ${ }^{17}$ La complementariedad de sus investigaciones

\footnotetext{
15 Véase VENN, C. "Neoliberal Political Economy, Biopolitics and Colonialism. A Transcolonial Genealogy of Inequality”, Theory, Culture \& Society, Vol. 26(6), 2009, p. 209.

${ }^{16}$ La idea de "gran relato" refiere a la dimensión epocal del diagnóstico. Dicho concepto es utilizado por Bidet para identificar a aquellos discursos que hacen referencia a las largas duraciones y que, adoptando un enfoque totalizante, se dirigen al diagnóstico de la cuestión de "la totalidad social, considerada en términos de la "racionalidad occidental", BIDET, J. "Foucault y el liberalismo: Racionalidad, revolución, resistencia”, Argumentos, Año 19 No. 52, Septiembre-Diciembre, 2006, p. 13. Es decir, lo que en nuestra grilla epocal-topológica correspondería al análisis histórico de los sistemas de dominantes.

${ }^{17}$ Con la idea de que constituyen pensamientos inextricables Bidet expone un tipo de complementariedad que no busca destacar principalmente ni las huellas del pensamiento de Marx en el de Foucault ni los puntos en que ambos planteos convergen. Ante todo resalta el hecho de que la teoría foucaultiana al implicar la apertura hacia nuevos campos y objetos de investigación y problematización no debe ser considerada como un simple 'complemento' aportado a Marx, sino como el lugar donde ir a buscar "lo que falta en Marx", BIDET, J. "Foucault y el liberalismo: Racionalidad, revolución, resistencia", Argumentos, Año 19 No. 52, Septiembre-Diciembre, 2006, p. 25. Para una discusión acerca de diversas relaciones posibles y efectivas entre los pensamientos de Foucault y de Marx pueden destacarse: LEMKE, T., LEGRAND, S. et al. Marx y Foucault. Buenos Aires: Nueva Visión, 2006 y BALIBAR, E. "Foucault y Marx. La postura del nominalismo”, en: Balbier, E., Deleuze, G. et al., Michel Foucault filósofo. Barcelona: Gedisa, 1999, pp. 48-66. Sin embargo, es preciso dejar sentada nuestra posición al respecto,
} 
encontraría su punto de apoyo en la estructura de "gran relato" que tienen ambos discursos. Así, mientras que el "gran relato" marxiano, al centrarse en el estudio del capitalismo en relación con la cuestión del gobernarse, tendría como horizonte una política revolucionaria; el "gran relato" foucaultiano, girando en torno del análisis del liberalismo y de la relación entre gobernantes y gobernados, proyectaría prácticas de resistencia.

Este "gran relato" de Foucault -es decir, desde nuestra perspectiva, la dimensión epocal de un diagnóstico que recurre a la genealogía para hacer la historia de la gubernamentalidad- se desplegaría, entonces, a partir de la investigación genealógica del Estado moderno y la razón gubernamental y estaría compuesto, de acuerdo con

pues sólo en parte es convergente con la de Bidet. En primer lugar, es necesario tener en cuenta que en la misma entrevista en la que reveló que utilizaba y citaba a Marx sin ponerlo entre comillas, Foucault afirma lo siguiente: "Es imposible hacer historia actualmente sin recurrir a una serie interminable de conceptos, ligados directa o indirectamente al pensamiento de Marx, sin situarse en un horizonte que fue descrito y definido por Marx. En último término, uno se podría preguntar qué diferencia puede haber entre ser historiador y ser marxista.[...] Porque en el interior de este horizonte general, definido o codificado por Marx, comienza la discusión con aquellos que se declaran marxistas, pero que juegan esta especie de regla de juego que no es la del marxismo, sino la de la comunistología, es decir, la línea definida por los partidos comunistas que indican la manera en que se debe utilizar a Marx para ser declarado, por ellos, marxista". Ahora bien, es importante tener presente que inmediatamente después de este reconocimiento señala que con Nietzsche establece una relación análoga. En efecto, el proyecto foucaultiano tal como lo concebimos en este trabajo se inscribe entre las coordenadas marxianas y nietzscheanas, pues "Nietzsche fue quien propuso como blanco esencial, digamos al discurso filosófico, la relación de poder [, m]ientras que para Marx, la apuesta eran las relaciones de producción", véase, FOUCAULT, M. "Entrevista sobre la prisión: el libro y su método", en: Estrategias de poder. Obras esenciales, Volumen II. Barcelona: Paidós, 1999, pp. 311-312. En este sentido, y en segundo lugar, cabe subrayar que, en sintonía con la propuesta de Bidet, la grilla bélico-gubernamental bien puede interpretarse como aquello que falta en Marx y en el marxismo, pues tal como señala Foucault, por un lado, la guerra ha sido insuficientemente pensada en dicha tradición: "lo que me sorprende, de los análisis marxistas, es que siempre se trata en ellos de 'la lucha de clases', pero hay en esta expresión una palabra a la que se presta, sin embargo, menor atención, y es la de 'lucha'. Ahí conviene matizar. Los grandes entre los marxistas (empezando por Marx) han insistido mucho en los problemas 'militares' (Ejército como aparato de Estado, levantamiento armado, guerra revolucionaria). Pero cuando hablan de 'lucha de clases' como resorte general de la Historia, se preocupan, sobre todo, de saber qué es la clase, dónde se sitúa, a quien engloba, pero nunca qué es concretamente la lucha. Con una excepción más o menos: los textos no teóricos, sino históricos, del propio Marx, que son mucho más sutiles”, véase FOUCAULT, M. “¿Qué es la crítica? (Crítica y Aufklärung), Daimon, revista de filosofía. Murcia, n 11, 1995, p. 129; mientras que por el otro, la cuestión del gobierno no sólo no ha sido suficientemente pensada por el marxismo, sino que debiera ser inventada ya que "la importancia del texto en el socialismo está, me parece, a la altura misma de la laguna misma constituida por la falta de un arte socialista de gobernar", véase FOUCAULT, M. Seguridad, territorio, población, Buenos Aires, Fondo de Cultura Económica, 2006, 119-120. Sin embargo, en tercer lugar, es necesario subrayar que la grilla epocal-topológica se constituye en el horizonte abierto por la distancia respecto de la concepción dialéctica de análisis vinculada a una temporalidad lineal y teleológica, determinada en última instancia económicamente, pues esta grilla epocal-topológica permite analizar la larga duración pero a través de una lógica estratégica capaz de interrogar la relación contingente entre tecnologías heterogéneas. En cuarto y último lugar, vale destacar como justificación, quizás, para una nota tan extensa- la indicación vertida por nuestro autor al final del curso de 1978 cuando se refiere a la utilización que hacia fines de siglo XVI se hacía de Maquiavelo: "desde este punto de vista, nuestro Maquiavelo es Marx: aunque la cosa no pase por él, se dice a través de él” FOUCAULT, M. Seguridad, territorio, población. Buenos Aires, Fondo de Cultura Económica, 2006, p. 285. 
Bidet, por tres momentos progresivos que definirían su "apuesta política" última en términos de la sobrevivencia del capitalismo a través de la posibilidad de invención de un nuevo capitalismo. ${ }^{18}$ Estos momentos son la soberanía, i.e. el Estado de justicia, en el Renacimiento; la disciplina, i.e. el Estado administrativo, que signa la época de la Razón de Estado a partir de la paz de Westfalia e inaugura la modernidad política; el gobierno, i.e. la gubernamentalización del Estado, que a partir de la fisiocracia se centra en la economía política y la gestión de la población qua sujeto colectivo diferente al del contrato social.

Es cierto que Bidet nota que este "gran relato" está estructurado a partir de la sucesión de épocas aunque, simultáneamente, sea presentado como un triángulo de tecnologías heterogéneas. Sin embargo, afirma que en el curso Nacimiento de la biopolítica esta heterogeneidad habría sido "reducida" a partir de la presentación del neoliberalismo como un orden jurídico-económico y haciendo a un lado la dimensión disciplinaria. Esto sería así hasta el final del curso, pues allí Foucault propondría interpretar -y Bidet considera esto como la "última lección a extraer de su enseñanza"los tres elementos del triángulo a partir de la idea de juego entre tres artes de gobernar: gobernar en la verdad, en la Razón de Estado, en la racionalidad de los gobernados. ${ }^{19}$ En efecto, para Bidet, a pesar de que Foucault privilegie al liberalismo, su enfoque general lo desborda por completo en la medida en que las políticas nacionalistas, estatales e incluso el marxismo, participarían del debate político en torno de la gubernamentalidad.

Sin embargo, el comentador sostiene que Foucault, al tomar como punto de partida el concepto de 'arte de gobernar' -i.e. la problematización de la relación entre gobernantes y gobernados-, evitaría "la cuestión, revolucionaria, del derecho considerado a partir del problema de su 'comienzo', o sea, 'el problema teórico y jurídico de la constitución originaria de la sociedad'”. ${ }^{20}$ En efecto, esto daría lugar a la idea foucaultiana de una política de resistencias a partir del concepto de libertad entendido como la relación actual entre gobernantes y gobernados. La perspectiva foucaultiana quedaría, así, expuesta a la crítica que subraya cierto reduccionismo de la cuestión política moderna a la cuestión negociable entre gobernantes y gobernados en

\footnotetext{
${ }^{18}$ Véase FOUCAULT, M. Seguridad, territorio, población. Buenos Aires, Fondo de Cultura Económica, 2006, p. 16.

${ }^{19}$ Véase FOUCAULT, M. Seguridad, territorio, población. Buenos Aires, Fondo de Cultura Económica, 2006, p. 20.

${ }^{20}$ BIDET, J. "Foucault y el liberalismo: Racionalidad, revolución, resistencia”, Argumentos, Año 19 No. 52, Septiembre-Diciembre, 2006, p. 22.
} 
términos de la dicotomía -que se juega en el espacio de la sociedad civil- entre sujeto económico y sujeto de derecho. Bidet señala al respecto que "en realidad, más que esta pareja dudosa, la figura del 'triángulo' podría haberle conducido a identificar mejor la naturaleza del desafío político moderno: el de una subjetividad política" ${ }^{21}$ es decir, la cuestión identitaria del nosotros como sujeto del gobernarse. De ahí la sugerencia del comentador acerca de que tanto la resistencia como la revolución deberían "leerse mutuamente en el espejo de la subjetivación". ${ }^{22}$ En efecto, la cuestión soberana de la ciudadanía y la legitimidad y la disciplinaria de la organización y la administración deberían ser tenidas también en cuenta si se pretende abordar la cuestión de la resistencia y la revolución en el marco del triángulo de las artes de gobernar heterogéneas. En este sentido, Bidet concluye lo siguiente:

Lo importante no es saber en qué medida Foucault se adhiere a los liberalismos de los que habla. Lo importante es más bien, de una parte, su reinterpretación de 'nuestra racionalidad política' moderna en términos de relaciones entre gobierno y gobernados, alternativa a la pretensión revolucionaria de gobernarse. Pero es también la positividad del saber-poder dado a la gubernamentalidad moderna, y su contraparte según la cual el ciudadano supuestamente soberano no escapa tampoco a la sujeción a saberes y poderes. ${ }^{23}$

\section{El triángulo de la racionalidad política moderna: heterogeneidad y}

\section{dominancia.}

Frente a la hipótesis de un "gran relato" foucaultiano modulado en tres paradigmas epocales sucesivos, Collier resalta la emergencia, hacia 1978, de un modelo de análisis basado en la heterogeneidad de los elementos constitutivos del triángulo.

Es conocida la tipificación foucaultiana de diagramas de poder sucesivos ${ }^{24}$ a partir de la elaboración de tres diferentes paradigmas: el de soberanía, el de disciplina y el de seguridad o gobierno. Sin embargo, en Seguridad, territorio, población la explicación de su relación introduce una modificación clave respecto de la comprensión

\footnotetext{
${ }^{21}$ BIDET, J. "Foucault y el liberalismo: Racionalidad, revolución, resistencia”, Argumentos, Año 19 No. 52, Septiembre-Diciembre, 2006, p. 22.

${ }^{22}$ BIDET, J. "Foucault y el liberalismo: Racionalidad, revolución, resistencia", Argumentos, Año 19 No. 52, Septiembre-Diciembre, 2006, p. 27.

${ }^{23}$ FOUCAULT, M. Seguridad, territorio, población. Buenos Aires, Fondo de Cultura Económica, 2006, p. 26.

${ }^{24}$ Sobre la explicación foucaultiana en términos de sucesión y paulatino reemplazo. Véase FOUCAULT, M. El poder psiquiátrico. Buenos Aires: Fondo de Cultura Económica, 2005, p. 62 y ss. y FOUCAULT, M. Vigilar y castigar. México: Siglo Veintiuno editores, 1999, 77 y ss. y 139 y ss.
} 
anterior, realizada en términos de sucesión temporal. Es sabido que Foucault procede en sus análisis a partir de grandes bloques paradigmáticos dicotómicos que le permiten señalar la especificidad de cada uno de ellos ${ }^{25}$. No obstante, esta distinción es analítica, pues en la historia nunca aparecen en su forma pura ni tan claramente separados, sino que, antes bien, dichos paradigmas se combinan y conviven de diferentes modos según el período histórico estudiado. Por lo tanto, en sentido estricto, no deberían identificarse los períodos históricos con determinadas figuras paradigmáticas de ejercicio del poder. Aún así, la distinción paradigmática permanecería útil para captar el modo de la combinación de dispositivos heterogéneos. Respecto de esto en la clase del 11 de enero de 1978 Foucault dice lo siguiente:

No tenemos de ninguna manera una serie en la cual los elementos se suceden unos a otros y los que aparecen provocan la desaparición de los precedentes. No hay era de lo legal, era de lo disciplinario, era de la seguridad. [...] lo que va a cambiar es sobre todo la dominante, o más exactamente, el sistema de correlación entre los mecanismos jurídico legales, los mecanismos disciplinarios y los mecanismos de seguridad. ${ }^{26}$

En consecuencia, no sólo cambian los mecanismos sino, sobre todo, el lugar que ocupan en la nueva economía de poder. Según interpreta Collier, Foucault se desplazaría de un estilo de diagnóstico -que enfatizaría la sistematicidad y la coherencia funcional- hacia otro que se centraría en los sistemas de correlación entre los diferentes mecanismos. El comentador se refiere a esta nueva forma de indagación con el concepto de "análisis topológico del poder". ${ }^{27}$

En efecto, Foucault distingue entre la historia de las técnicas -por ejemplo, de la técnica celular (disciplinaria) o de la estadística criminal (técnica de seguridad)- que tiene por objetos de estudio su emergencia, sus desplazamientos, su utilización, los conflictos que suscita su implementación, etc., y la historia de las tecnologías, es decir:

La historia mucho más global, pero desde luego también mucho más vaga, de las correlaciones y los sistemas de dominantes que hacen que, en una sociedad dada y para tal o cual sector específico -pues las cosas no siempre van a evolucionar forzosamente al mismo ritmo en

\footnotetext{
${ }^{25}$ Por ejemplo, mientras que en Vigilar y castigar expone la especificidad de la tecnología disciplinaria a través del contrapunto con el mecanismo de soberanía, por el contrario en Seguridad, territorio, población, de la oposición entre el dispositivo de seguridad y la tecnología jurídico-disciplinaria se obtendrán las características principales del paradigma de gobierno.

${ }^{26}$ FOUCAULT, M. Seguridad, territorio, población. Buenos Aires, Fondo de Cultura Económica, 2006, p. 23, la itálica es nuestra

27 Véase COLLIER, S. "Topologies of Power. Foucault's Analysis of Political Government beyond 'Governmentality", Theory, Culture \& Society, Vol. 26(6), 2009, p. 88-90.
} 
uno $\mathrm{u}$ otro sector, en un momento, una sociedad o un país determinados-, se introduzca, por ejemplo, una tecnología de seguridad que hace suyos y pone en funcionamiento dentro de su propia táctica elementos jurídicos, elementos disciplinarios, y a veces llega a multiplicarlos. ${ }^{28}$

En relación con esto, consideramos que la oposición entre la lógica dialéctica y la lógica de la estrategia desarrollada en Nacimiento de la biopolítica permitirá comenzar a echar luz sobre el sentido de la distinción entre "las correlaciones y los sistemas de dominantes".

Mientras que, de acuerdo con Foucault, la lógica dialéctica procura hacer valer términos contradictorios en el elemento de lo homogéneo prometiendo su resolución en una unidad, por el contrario, la lógica estratégica establece conexiones posibles entre términos heterogéneos que permanecen heterogéneos. En efecto, la heterogeneidad, según Foucault, "nunca es un principio de exclusión o, si lo prefieren, la heterogeneidad jamás impide la coexistencia, ni la unión, ni la conexión" ${ }^{29}$ y es a partir de esto que se puede llevar a cabo un análisis que haga valer la lógica de la estrategia, es decir "la lógica de la conexión de lo heterogéneo y no la lógica de la homogeneización de lo contradictorio". 30

Apoyándose en estas referencias, Collier afirma que hay un quiebre fundamental en la obra de Foucault: desde la búsqueda de un diagnóstico global y epocal todavía presente en Defender la sociedad y en La voluntad de saber -que estarían aún en la línea de Vigilar y castigar - hacia el nuevo modelo topológico de análisis desplegado en los cursos de 1978 y 1979. ${ }^{31}$ No obstante, según el comentador, la ruptura no puede ser captada si se fija la atención en la categoría de 'gubernamentalidad' como el concepto clave, pues Foucault la define de modo tal que no sólo da la idea de una secuencia temporal, sino que también afirma que "vivimos en la era de la gubernamentalidad,

\footnotetext{
${ }^{28}$ FOUCAULT, M. Seguridad, territorio, población. Buenos Aires, Fondo de Cultura Económica, 2006, p. 24, la itálica es nuestra.

${ }^{29}$ FOUCAULT, M. Nacimiento de la biopolítica. Buenos Aires, Fondo de Cultura Económica, 2007, p. 62.

${ }^{30}$ FOUCAULT, M. Nacimiento de la biopolítica. Buenos Aires, Fondo de Cultura Económica, 2007, p. 62. Sobre el concepto de estrategia en la obra de Foucault remitimos al tratamiento de Nosetto donde distingue tres modos de entender la conexión de los elementos heterogéneos (instrumental, lúdica y bélica) y dos tipos de estrategias: las de poder y las de lucha. Véase NOSETTO, L. Foucault y la política. San Martín, Universidad Nacional de San Martín. USAM-EDITA, 2013.

${ }^{31}$ Véase COLLIER, S. "Topologies of Power. Foucault's Analysis of Political Government beyond 'Governmentality”, Theory, Culture \& Society, Vol. 26(6), 2009, p. 85-87.
} 
descubierta en el siglo XVIII", ${ }^{32}$ En sentido estricto, para el comentador, el concepto de gubernamentalidad designaría el género (diagramas de racionalidad política) del cual las racionalidades políticas específicas tales como el neoliberalismo serían una especie. ${ }^{33}$ En consecuencia, el desplazamiento habría de ser explicado a partir de la exposición de la lógica del análisis topológico del poder antes que a partir del recurso a la creación de la grilla gubernamental.

Es necesario detenerse aquí un momento y ahondar en el argumento de Collier. El punto de partida es el concepto de bio-poder tal como aparece en el curso y el libro de 1976. Allí Foucault señala el tránsito desde el poder de soberanía hacia el bio-poder, es decir, desde la era de la soberanía a la era del bio-poder y la sociedad de normalización. Esto se explicaría, según Collier, en términos cuasi-funcionalistas por referencia al contexto más amplio del desarrollo del capitalismo, la explosión demográfica, la expansión de los mercados, la urbanización, etc., ante los cuales el viejo modelo de soberanía se mostraba incapaz de hacerles frente. Así, como afirma Foucault, el capitalismo "no pudo afirmarse sino al precio de la inserción controlada de los cuerpos en el aparato de producción y mediante el ajuste de los fenómenos de población a los procesos económicos". ${ }^{34}$ Siguiendo esta línea argumental el concepto de bio-poder permitiría una explicación en términos de cierta complementariedad funcional entre la disciplina del cuerpo individual y la biopolítica de las poblaciones. El ejemplo paradigmático de este caso en el que disciplina y biopolítica confluyen, lo representaría el dispositivo de sexualidad a través del cual "el sexo es, a un tiempo, acceso a la vida del cuerpo y a la vida de la especie". ${ }^{35}$ Pero también es ejemplo de esta confluencia la ciudad obrera tal como la describe Foucault en Defender la sociedad (2000a: 227). En efecto, Foucault fundamenta esta convergencia y articulación entre el poder disciplinario y los controles regularizadores en el hecho de que se tratan de mecanismos de distinto nivel de aplicación. ${ }^{36}$ Así, ellos constituirían dos polos o dimensiones de un

\footnotetext{
${ }^{32}$ FOUCAULT, M. Seguridad, territorio, población. Buenos Aires, Fondo de Cultura Económica, 2006, p. 137, la itálica es nuestra.

33 COLLIER, S. "Topologies of Power. Foucault's Analysis of Political Government beyond 'Governmentality”, Theory, Culture \& Society, Vol. 26(6), 2009, p. 99.

${ }^{34}$ FOUCAULT, M. Historia de la sexualidad I: La voluntad de saber. México: Siglo Veintiuno editores, 2000, p. 170.

${ }^{35}$ FOUCAULT, M. Historia de la sexualidad I: La voluntad de saber. México: Siglo Veintiuno editores, 2000, p. 176.

${ }^{36} \mathrm{La}$ biopolítica es "una tecnología de poder que no excluye la primera, que no excluye la técnica disciplinaria sino que la engloba, la integra, la modifica parcialmente y, sobre todo, que la utilizará implantándose en cierto modo en ella, incrustándose, efectivamente, gracias a esta técnica disciplinaria previa. Esta técnica no suprime la técnica disciplinaria, simplemente porque es de otro nivel, de otra
} 
único proceso general de normalización ${ }^{37}$ que opera disciplinando, optimizando y controlando la vida de los individuos y las poblaciones. De acuerdo con Collier este proceso de normalización provee una "resolución funcional" de las tensiones previas planteadas a partir de la emergencia de la sociedad capitalista y de las demandas de organizar las poblaciones de acuerdo a los procesos económicos y de insertar los cuerpos individuales en el aparato de producción. No se trataría, entonces, de un análisis topológico del poder, sino de uno cuasi-funcionalista, totalizante y epocal que haría foco en la sistematicidad y la coherencia antes que en la heterogeneidad. De esta forma es que Collier arriba a sus conclusiones respecto del desplazamiento metodológico de Foucault: mientras que en Vigilar y castigar se analiza el tránsito de la soberanía a la disciplina y en Defender la sociedad y La voluntad de saber de la soberanía al biopoder -donde éste es concebido como la suma del poder disciplinario y del poder regulador o biopolítico-, por el contrario, a partir de Seguridad, territorio, población emerge la serie heterogénea soberanía-disciplina-seguridad. ${ }^{38}$ En consecuencia, se pasaría de una lógica inmanente, simplista y unitaria del desarrollo del poder a una lógica de la estrategia en la que la soberanía no sería simplemente reemplazada por el bio-poder. Se trataría, antes bien, de una lógica en la que la emergencia de los dispositivos de seguridad implica la reactivación y transformación de ciertos elementos del poder soberano, dando lugar a un sistema de correlación entre los mecanismos jurídico-legales, los disciplinarios y los de seguridad. No se trataría pues, en adelante, de transformaciones epocales que tienen lugar con cierta inevitabilidad impersonal, sino del análisis de los procesos de recombinación a través de los cuales las tecnologías de poder son redistribuidas. En efecto, la indagación pasaría de estar centrada en la sucesión temporal para hacer foco en el problema espacial del lugar que ocupan los elementos en la serie heterogénea. ${ }^{39}$

Si bien podemos acordar con el argumento general presentado por Collier, no obstante, debemos remarcar su unilateralidad. El autor parece consciente de ello cuando hace notar que a través de este análisis topológico se corre el riesgo de enfatizar en

escala, tiene otra superficie de sustentación y se vale de instrumentos completamente distintos". FOUCAULT, M. Defender la sociedad. Buenos Aires: Fondo de Cultura Económica, 2000, p. 219.

37 "La sociedad de normalización es una sociedad donde se cruzan, según una articulación ortogonal, la norma de la disciplina y la norma de la regulación". FOUCAULT, M. Defender la sociedad. Buenos Aires: Fondo de Cultura Económica, 2000, p. 229.

${ }^{38}$ FOUCAULT, M. Nacimiento de la biopolítica. Buenos Aires, Fondo de Cultura Económica, 2007, p. 87.

39 Véase COLLIER, S. "Topologies of Power. Foucault's Analysis of Political Government beyond 'Governmentality”, Theory, Culture \& Society, Vol. 26(6), 2009, p. 93. 
exceso la contingencia de determinadas formaciones específicas, a expensas del desarrollo de una perspectiva más amplia sobre los determinados diagramas de poder perspectiva que permitiría hacer un diagnóstico global sobre una época determinada-. ${ }^{40}$

Desde nuestro punto de vista, la unilateralidad de la interpretación de Collier reside en la atención prestada exclusivamente al concepto de "sistemas de correlación" en desmedro de aquel otro al que está unido en las dos citas consignadas anteriormente: el de "sistemas de dominantes". En efecto, si el concepto de heterogeneidad y de sistema de correlaciones remite a la lógica de la estrategia y a la dimensión espacial y sincrónica del triángulo soberanía-disciplina-gobierno, en contrapartida, el concepto de sistema de dominantes parece referir tanto a la articulación funcional interna de los elementos del triángulo, como a la dimensión diacrónica que permite explicar las diferentes configuraciones en momentos sucesivos. Precisamente, de una época a otra los elementos dominantes $-\mathrm{y}$, por ello, determinantes de la especificidad de un diagrama que es resultado de una combinación contingente de elementos heterogéneos- pasan a ser funciones secundarias y complementarias en un nuevo diagrama estructurado a partir de otra dominante. Esto explica por qué Foucault se refiere, por ejemplo, a la tecnología de seguridad, qua elemento dominante, diciendo que ésta incluye en su propia táctica, es decir como elementos subordinados, a los componentes disciplinarios y jurídicos. Por lo tanto, en este triángulo las relaciones varían según la dominante y éste es el modelo que permite captar las diferentes distribuciones de los mecanismos de poder, a través de la lógica de la estrategia, en los diversos momentos históricos de las sociedades.

Si por un lado, mediante el contraste, se obtienen los rasgos que definen cada uno de los paradigmas, por otro, a partir de dichos rasgos, de su supervivencia, cambio, subordinación de funciones se podrá determinar -según las maneras de la combinaciónqué tecnología de poder es la dominante en cada época, y a partir de esto, qué tipo de distribución de las tecnologías de poder da el tono específico a las relaciones de poder formando un diagrama correspondiente a un momento histórico. En consecuencia, ambos sistemas se requieren recíprocamente para llevar a cabo un diagnóstico global y singular a la vez, atento tanto a la dominante que da la tónica a las relaciones de poder en una época, sociedad o coyuntura determinadas, cuanto a la contingencia a partir de la

40 Véase COLLIER, S. "Topologies of Power. Foucault's Analysis of Political Government beyond 'Governmentality”, Theory, Culture \& Society, Vol. 26(6), 2009, p. 105-106. 
cual estos elementos coexisten permaneciendo heterogéneos. Tener en cuenta sólo la dimensión topológica del análisis es enfatizar en exceso la contingencia perdiendo de vista los desplazamientos globales. Por su parte, el riesgo de enfocar sólo la dimensión histórica secuencial es lo que Collier denomina, siguiendo a Rose, "rigidificación". ${ }^{41}$ En este último caso el riesgo reside en terminar por concebir la secuencia histórica en términos de una teleología o un destino inexorable en el que se van desplegando coherente, necesaria y sistemáticamente diagramas de poder que reemplazan a los anteriores. Se advierte, por lo tanto, cómo la consideración conjunta de las correlaciones y los sistemas de dominante que proponemos permite conjurar estos dos peligros teniendo en cuenta tanto la lógica estratégica que explica topológicamente la convivencia de las tecnologías de poder cuanto la variación histórica relativa al dominio de una de ellas sobre las demás. El concepto de gubernamentalidad como "generalidad singular" daría cuenta de esta doble dimensión analítica. ${ }^{42}$

En este sentido, el desplazamiento no implicaría el abandono en la medida en que el paso de la soberanía a la disciplina y luego al gobierno no se daría en la forma del simple reemplazo de unos elementos por otros, sino en la del cambio en el sistema de correlación en el que una tecnología de poder determinada establece una relación de dominancia respecto de las otras, que pasarán a ser desde entonces para esa sociedad, ese país y en esa coyuntura, elementos subordinados tácticamente a dicha tecnología. En efecto, a lo largo de las genealogías foucaultianas los dos registros de análisis y diagnóstico -i.e. el epocal y el topológico- se despliegan simultáneamente. Esto explica la presencia conjunta de un análisis epocal orientado a la larga duración ${ }^{43}$ y de un abordaje topológico centrado en una coyuntura, un país o una sociedad específica ${ }^{44}$.

\footnotetext{
41 COLLIER, S. "Topologies of Power. Foucault's Analysis of Political Government beyond 'Governmentality", Theory, Culture \& Society, Vol. 26(6), 2009, p. 105.

${ }^{42}$ Véase SENELLART, M. "Situación de los cursos", en: Foucault, M., Seguridad, territorio, población. Buenos Aires: Fondo de Cultura Económica, 2006 pp. 449-451.

${ }^{43}$ En este sentido, véase Duarte, quien -a los fines de estudiar la problematización foucaultiana sobre la modernidad, para ponerla en relación con las de Heidegger y Arendt- sostiene lo siguiente: "Foucault no se limita al análisis historiográfico meticuloso de discursos y acontecimientos factuales, localizados y discontinuos, visto que él también procura establecer un sentido epocal de aquellas prácticas y discursos, presentando sus efectos duraderos a lo largo de la modernidad hasta nuestro presente". DUARTE, A. Vidas em risco. Crítica do presente em Heidegger, Arendt e Foucault. Rio de Janeiro: Gen-Forense Universitaria, 2010, p. 71, la traducción es propia.

${ }^{44}$ En este sentido, puede mencionarse el análisis topológico y coyuntural llevado a cabo durante la conferencia Nacimiento de la medicina social del año 1974 en la que Foucault expone el proceso de medicalización alemán de comienzos del siglo XVIII, francés de finales del mismo siglo e inglés del siglo XIX. Véase FOUCAULT, M. "Nacimiento de la medicina social", en: Estrategias de poder. Obras esenciales, Volumen II. Barcelona: Paidós, 1999 pp. 363-384.
} 
Desde nuestra perspectiva, el "gran relato" epocal se articularía a través del concepto de sistema de dominante, con los patrones de correlación y el análisis topológico. Sin embargo, según consideramos, esto no ocurriría desde que Foucault lo explicita en Seguridad, territorio, población sino, cuanto menos, desde que en Vigilar y castigar el discurso jurídico soberano del contrato coexiste, manteniendo su heterogeneidad, con el funcionamiento de la tecnología disciplinaria. ${ }^{45}$ Una aproximación a los textos anteriores a 1978 con una grilla que no acentúe unilateralmente la coherencia funcional permite encontrar, también en ellos, las tensiones entre elementos heterogéneos. Por ejemplo, el concepto de racismo de Estado implica la combinación del poder de soberanía con el bio-poder tanto en La voluntad de saber como en Defender la sociedad aun cuando permanecen siendo heterogéneos. ${ }^{46}$ En efecto, al contrario de lo que sugiere Collier -cuando se refiere a la coherencia sistemática con que disciplina y biopolítica parecen articularse en La voluntad de saber y en Defender la sociedad-, puede sostenerse que en el caso del racismo -que en el análisis Collier es omitido- soberanía, guerra y bio-poder se despliegan conjuntamente manteniendo su heterogeneidad, lo cual no impide su coexistencia ni su articulación instrumental. La idea de heterogeneidad está presente en los análisis foucaulteanos, al menos desde 1975, así como el diagnóstico epocal permanece aún después de 1978, pues de otro modo, no podría explicarse que en Seguridad, territorio, población -en el transcurso del mismo argumento- Foucault sostenga que "es preciso comprender las cosas no como el reemplazo de una sociedad de soberanía por una sociedad de disciplina y luego de una sociedad de disciplina por una sociedad, digamos, de gobierno. De hecho, estamos ante un triángulo: soberanía, disciplina y gestión gubernamental" ${ }^{47}$ y luego afirme que el proceso de 'gubernamentalización' del Estado marca "la era de la gubernamentalidad, descubierta en el siglo XVIII". ${ }^{48}$

Por lo tanto, el diagnóstico según el cual se pasa de la dominancia de la soberanía a la de la disciplina y luego a la de la gestión gubernamental permite explicar este tránsito tanto en términos de épocas o momentos históricos determinados -

\footnotetext{
${ }^{45}$ Véase FOUCAULT, M. Vigilar y castigar. México: Siglo Veintiuno editores, 1999, p. 225.

${ }^{46}$ Véase FOUCAULT, M. Historia de la sexualidad I: La voluntad de saber. México: Siglo Veintiuno editores, 2000, pp. 178-181 y FOUCAULT, M. Defender la sociedad. Buenos Aires: Fondo de Cultura Económica, 2000, pp. 232-233.

${ }^{47}$ FOUCAULT, M. Seguridad, territorio, población. Buenos Aires, Fondo de Cultura Económica, 2006, p. 135.

${ }^{48}$ FOUCAULT, M. Seguridad, territorio, población. Buenos Aires, Fondo de Cultura Económica, 2006, p. 137.
} 
desplegados sucesivamente en una temporalidad de larga duración- cuanto en términos de elementos heterogéneos articulados según sistemas o patrones de correlación contingentes que constituyen un triángulo, es decir, una singularidad topológica en la que los elementos se relacionan permaneciendo heterogéneos. Consecuentemente, los sistemas de dominante permiten explicar tanto los sistemas de correlación al interior de un triángulo en el que a la tecnología dominante se le subordinan las demás, cuanto la denominación epocal que se corresponde con la hegemonía de tal tecnología dominante. Es por esto que consideramos que simultáneamente debe comprenderse el discurso foucaultiano como un "gran relato" -que hace funcionar una grilla epocal a través de la cual el análisis de los sistemas de dominante permite exponer los debates y los combates a través de los cuales el vértice gubernamental del triángulo devino hegemónico, marcando nuestra actualidad como tal- y como un análisis estratégico de la conexión de elementos heterogéneos -llevada a cabo a través de un análisis topológico que hace posible comprender las correlaciones contingentes y coyunturales que explican la forma en que la tecnología de seguridad se apropia y pone en funcionamiento dentro de su propia táctica los elementos jurídicos y disciplinarios que se le subordinan-.

\section{Consideraciones finales.}

El debate y la posterior articulación de las perspectivas de Bidet y Collier me ha permitido argumentar en favor de la idea de una grilla de inteligibilidad simultáneamente epocal y topológica, orientada por una lógica de la estrategia, como marco para comprender la historia política de la gubernamentalidad en los cursos intermedios de Foucault. De este modo, se ha puesto en consideración la articulación entre de sistema de correlación y de sistema de dominante para establecer la dinámica que rige la configuración de las relaciones de poder bajo la figura del triángulo soberanía -disciplina -gobierno. El proceso de gubernamentalización del Estado y su progresiva convergencia -que encuentra en el neoliberalismo su plena realización- con la idea de Estado de Derecho y con la autoconstitución de los sujetos como empresarios marca una relación contingente entre gobierno (economía política y población), soberanía y disciplina. En efecto, el concepto de heterogeneidad que se encuentra a la base de la interpretación estratégica de las relaciones de poder permite pensar cada uno de sus elementos como tecnologías de poder que permanecen heterogéneas, es decir, cuyo sistema de correlación no es fruto una complementariedad necesaria, sino del juego de arreglos y compromisos que sostienen una determinada forma de vincular al 
derecho y la economía con un forma particular de concebir sujeto. La serie Estado de derecho-mercado de competencia perfecta- población económica (constituida por sujetos empresarios de sí mismos) al no ser concebida como un sistema cerrado en el que por fin convergen coherentemente los tres elementos del triángulo, sino como un espacio en el que en una coyuntura determinada históricamente esta serie se articula de manera contingente permite suponer no sólo la convergencia y complementariedad entre las tecnologías de poder, sino también las tensiones y disputas que su coexistencia heterogénea también supone. De este modo, no sólo soberanía y disciplina aparecen en la historia de la gubernamentalidad como elementos subordinados al gobierno en el sistema de correlación en el que éste domina el triángulo de las tecnologías de poder, sino que también constituyen puntos de apoyo para la resistencia y la impugnación de tal sistema de correlación. Es por eso que la crítica de la soberanía y el Estado de Derecho, pero también la crítica de la economía política y de la constitución del sujeto poblacional pueden devenir la condición para pensar cierta forma de resistencia apoyada en otro concepto de derecho, otra relación económica y otra forma de subjetivación, todo ello en el marco de un pensamiento de otra forma de ser gobernado.

\section{Referencias:}

BALBIER, E., DELEUZE, G. et al. ; Michel Foucault filósofo, Barcelona, Gedisa, 1999.

BALIBAR, E. "Foucault y Marx. La postura del nominalismo", en: Balbier, E., Deleuze, G. et al., Michel Foucault filósofo. Barcelona: Gedisa, 1999, pp. 48-66.

BIDET, J. "Foucault y el liberalismo: Racionalidad, revolución, resistencia", Argumentos, Año 19 No. 52, Septiembre-Diciembre, 2006, pp. 1-27

CASTRO, E. El vocabulario de Michel Foucault, Buenos Aires, UNQUI-Prometeo, 2004.

CASTRO, E. Lecturas foucaulteanas. Una historia conceptual de la biopolítica, La Plata, UNIPE, Editorial Universitaria, 2011.

CASTRO-GÓMEZ, S. Historia de la gubernamentalidad. Razón de Estado, liberalismo y neoliberalismo en Michel Foucault. Bogotá: Siglo del Hombre Editores, 2012.

COLLIER, S. "Topologies of Power. Foucault's Analysis of Political Government beyond 'Governmentality”, Theory, Culture \& Society, Vol. 26(6), 2009, pp. 78-108.

DONNELLY, M. "Sobre los diversos usos de la noción de biopoder", en: Balbier, E., Deleuze, G. et al., Michel Foucault filósofo. Barcelona: Gedisa, 1999, pp. 193-197. 
DUARTE, A. Vidas em risco. Crítica do presente em Heidegger, Arendt e Foucault. Rio de Janeiro: Gen-Forense Universitaria, 2010.

FOUCAULT, M. “¿Qué es la crítica? (Crítica y Aufklärung), Daimon, revista de filosofía. Murcia, $\mathrm{n}^{\circ}$ 11, 1995, pp. 5-24.

FOUCAULT, M. "Entrevista sobre la prisión: el libro y su método", en: Estrategias de poder. Obras esenciales, Volumen II. Barcelona: Paidós, 1999, pp. 297-312.

FOUCAULT, M. "Nacimiento de la medicina social", en: Estrategias de poder. Obras esenciales, Volumen II. Barcelona: Paidós, 1999, pp. 363-384.

FOUCAULT, M. Defender la sociedad. Buenos Aires, Fondo de Cultura Económica: 2000.

FOUCAULT, M. Dits et Écrits II. Paris: Gallimard, 2001.

FOUCAULT, M. El poder psiquiátrico. Buenos Aires: Fondo de Cultura Económica, 2005.

FOUCAULT, M. Historia de la sexualidad I: La voluntad de saber. México: Siglo Veintiuno editores, 2000.

FOUCAULT, M. Nacimiento de la biopolítica. Buenos Aires, Fondo de Cultura Económica, 2007.

FOUCAULT, M. Seguridad, territorio, población, Buenos Aires, Fondo de Cultura Económica, 2006.

FOUCAULT, M. Vigilar y castigar. México: Siglo Veintiuno editores, 1999.

LE BLANC, Guillaume; El pensamiento Foucault. Traductor Horacio Pons, Buenos Aires, Amorrortu, 2006.

LEMKE, T., LEGRAND, S. et al. Marx y Foucault. Buenos Aires: Nueva Visión, 2006.

NOSETTO, L. Foucault y la política. San Martín, Universidad Nacional de San Martín. USAM-EDITA, 2013.

REVEL, J. Dictionnaire Foucault. París: Ellipses, 2008.

SENELLART, M. "Situación de los cursos", en: Foucault, M., Seguridad, territorio, población. Buenos Aires: Fondo de Cultura Económica, 2006 pp. 417-453.

VENN, C. "Neoliberal Political Economy, Biopolitics and Colonialism. A Transcolonial Genealogy of Inequality”, Theory, Culture \& Society, Vol. 26(6), 2009, pp. 206-233.

VENN, C. y TERRANOVA, T. "Introduction. Thinking after Michel Foucault", Theory, Culture \& Society, 2009, Vol. 26(6):1-11. 
Recebido em junho de 2016

Aceito em dezembro de 2016

Luis Félix Blengino

UNLaM - UBA

lblengino@hotmail.com

Ciudad Autónoma de Buenos Aires, Argentina. 